\title{
Study on the Beauty of Texture Displayed by Materials in Modern Ceramic Art
}

\section{Chunru Zhao}

Xi’an University, Xi’an, Shaanxi, 710065

Keywords: texture beauty; materials beauty; ceramic art

\begin{abstract}
The ceramic texture has a wide range of performance and a wealth of potential, which can be called a piano in the construction of environmental art. It can be gorgeous or elegant, simple and crystal-clear, and the artistic effect it produces can be either to the "masses" or "subtleties." But despite their dedication to such abundance, their inherent qualities and potentials have not been valued and developed. New environments, new ideas, and new requirements are forcing us to reflect on traditional old ideas.
\end{abstract}

\section{Introduction}

The use of different muscle effects can create a variety of muscular beauty. Muscle effect and beauty of beauty are the language of ceramic art, and it is an indispensable arts and crafts method for all kinds of products made of ceramics. The surface of any product has its own special texture changes, and the aesthetics of this particular texture change are the artistic effects pursued by ceramic products. It can enrich the artistic value of porcelain works and improve its function of reviewing the beauty to make it more mature and beautiful.

Texture generally refers to the surface of the object to the visual or tactile organization, which includes material, letter and other content. Texture as a term of ceramic art, generally refers to the use of certain material materials, manufacturing methods and surface messenger organization caused by the firing stage in ceramic works. It is a comprehensive expression of factors such as material production and fire refining. In the works of art, the texture has two main forms: (1) Natural texture, which refers to the existence of a belief organization that does not exist in the artist's hands, such as: stone patterns, wood patterns, cloth patterns, or paper patterns. (2) Artificial texture refers to the texture tissue produced by the artist's human actions, such as brush strokes, ink marks, water marks, knife marks, and gouges. Since the ceramic sculpture needs to be fired to complete the entire production process after being molded by the artist, in terms of the artificiality of firing, the texture of the surface of the ceramic sculpture is an artificial texture, which is different from the ceramic art and other plastic arts. Department. The texture plays an important role in the creation and appreciation of ceramic sculptures. Of course, the material texture itself does not have aesthetic value. Its significance and value for art is not that it has its own stipulation, but that it is to create or appreciate the external projection of the inner reality of the subject, that is, the human aesthetic. Ideal, aesthetic sentiment, and outward projection of character sentiment [1]. The aesthetic significance and aesthetic value of the texture effect are completely determined by the person of the subject, depending on the communion and infiltration of its esthetic modality and the subject's conceptual emotion. Therefore, the aesthetic essence of texture lies in the emotional expression of the human's essential power, and the psychological mechanism that creates the aesthetic value of texture is the "heart with the same thing."

\section{Types of surface materials of ceramic materials}

Ceramic materials have a lot of muscle effects, and they have unique styles in art ceramics. The difference between art ceramics and other ceramic surface textures is: Other ceramics have certain adaptability and practicality. There are obvious differences in the design concept from the art ceramics' thinking and intention: The texture effect of artistic ceramics. The purpose is to increase it. The artistic appeal and spiritual essence of the arts fully embody the artistic features of various 
aspects, enabling viewers to more clearly understand the intentions of the works. However, if the surface of the ceramics is not changed, all the utensils are treated with the same surface treatment, which can only make people feel plain and boring, and also have no artistic impression and visual impact. The texture effect of ceramic materials, in general, there are prime fetus and glazing two kinds: The treatment of prime fetus has intaglio, positive carving, hollowing, stacking, tapping, squeezing, pressing, imprint, etc.; glazing with whole body glazing, local Glazing, glazing, enamel glaze, brush enamel, and a variety of color glaze combinations [2].

\section{The relationship between ceramics and muscle effect}

The artistic effects produced by an excellent and perfect ceramic art work cannot be separated from the perfect unity of style and decoration. If it is only a beautifully shaped and lacking decorative surface treatment, it is impossible to achieve the best artistic effect, or to focus only on Dealing with the surface and ignoring the perfection of the model can also not achieve a beautiful artistic expression. Judging from the design process, most of them have a surface texture after the first shape, and a certain shape should have the surface texture effect that is compatible with it. For example, when I created the color glaze comprehensive decorative tea set, “Arago Age”, I Purple gold glaze is used as a decoration for the mouth, the mouth, the mouth, and the underline, and the texture effect is created by using the engraving technique. The main body is decorated with a traditional blue-and-white pattern, making full use of the material's own characteristics, making the work perfect and full, organically combining the color glaze texture effect with traditional blue and white flowers, forming a strong rough and delicate contrast, with a unique artistic style and overall effect. Harmony blends, shines with each other, full of strong national characteristics. Colors are simple, classic and concise, with modern atmosphere, traditional and modern styles complement each other, with high practical value, reviewing the United States and collecting value [3].

Muscle effects are not only related to make-up, but also affect other aspects. For example, a cylindrical vase, if its surface is flat, will give people a feeling of a brighter upward, with a bouquet inserted, and it is also coordinated. If a parallel arrangement of mud bars is used to build the texture, then the texture of the mud The texture results in a rough and rustic feel. Patterns will also produce a sense of stability, and the rough textures and flowers will produce strong effects.

\section{Different effects of ceramic materials on muscle effect}

The inherent physical and chemical properties of material materials are often the determining factors of external physical beauty, so the appearance of the material can be characterized by the appearance of the material. For example, one of the precious varieties of Jingdezhen's traditional red glaze is "beauty drunk". Its color tone is different from that of blush, red rosary, lang kiln red and other copper-red glazes. In most cases, it is not a deep red but a kind of It is light red, resembling peach blossoms and jellyfish. Due to the burning atmosphere, the color of drunken beauty is ever-changing. Some have dark red spots densely in the pink of the cicada; some are dark red gradually faint into a light red tone around them; some are slightly yellowish or lighter in the lighter part than the crimson Green; Some appear in the shade of green with different blushes, and there is a curiosity about "the moss covered with peach blossoms." It is true that the change is multi-dimensional and the subtlety is infinite. The Qing Dynasty poet Hong Bei Jiang said: "If the spring is born in the spring, the beginning of the spring, and the red is like the morning and the sun," is a vivid depiction of the effect of this rich, poetic, glamorous, beautiful glaze [4].

For the first time in the modern ceramics creation, we should understand the uniqueness of different materials sensitively, and we must find a method that has the performance to express the beauty of the material. In recent years, the market has been gloriously decorated with glazed glazes. People are pursuing a leisure life that "goes back to nature and returns to their original nature". They like elegant colors, while pearl glazes have a light, calm, and attractive effect. . Painting on precious pearl glazes is like painting on Xuan paper, applying the rules of creation of Chinese paintings, and making the works to the best of their abilities. Different ceramic materials have great 
influence on the change of texture, and they have different treatment methods. Ceramics There are many kinds of soil products, but their plasticity is different. The plasticity of large soil can be used to beat, bend, pinch, push, squeeze, stir mud and other methods. Beating, that is, playing into a mud film, with a different texture of ceramic pat beat on the mud film, the effect appears in the expected; bending, that is hit into a mud film, then arbitrary bending, so that it has different texture effect; Extrusion is the use of mud or mud to squeeze randomly, so that it produces a variety of texture effects; pinching, refers to the production of pinched products, the petals, flower branches, with a pinch to form a picture, to create a beautiful Texture effect.

The plasticity of small soil can be handled by methods such as adhesion, air emptying and stacking. Bonding, the use of different sizes, different shapes of the mud, due to the size and density of the different bonding blocks, resulting in different effects; hollow, is in the surface of the body, dug holes in different shapes, due to the small hole Different arrangement results in different texture effects; accumulation, also in the shape of the surface of the pile or thick, ranging in size, different shapes, different texture results. The change of ceramic material is not only the change of plasticity, but also in the case of plasticity, due to the difference in color, the size of soil particles is different from the thickness of texture, and it also produces different muscle effects.

\section{The artistic expression of ceramic texture}

Excellent ceramic works of art, the resulting artistic effects, are inseparable from the aesthetic beauty and appearance of the decorative beauty. The unique beauty of ceramic art and the decorative beauty of texture should all be coordinated and indispensable. Perfect shape and no appearance of the texture treatment, it is impossible to achieve the best artistic effect. Or only pay attention to the appearance of the treatment effect, and ignore the perfection of the shape, the same can not achieve the ideal perfect artistic effect. From the initial design point of view, the general design process is to design the ceramic model first, and then to do the decorative treatment of the appearance of the ceramic model. This type of processing often leads to the design of one party moving to the other, and there will inevitably appear to be a passive design of one of the shapes and textures. Inevitably there will be one-sidedness in the design and texture design. Therefore, when designing and designing an artistic ceramic work, overall consideration should be given to the appearance of the shape and texture. Different ceramic stylings should be used to decorate with their coordinated external textures, or the appearance of textures is more conducive to embodying the inner beauty of the styling. For example: ceramic works with a more symmetrical range of appearance, the appearance of the texture can be relatively large changes in the clay paste, hollowing and other techniques [5]. Pottery clay paste can create a relief effect on the surface. Hollowing can make certain changes in the local shape of the model. In addition, intaglio can be used to sculpt irregular traditional decorative patterns. However, in the actual production, it is also possible to formulate the best styling and texture processing methods based on the texture, thickness, color, and the like of the existing ceramic materials. Making textures is the most common in ceramic decorative writing language. It refers to the theme of the producer's creation of a better performance. It has the intention to artificially create some textures in the work, serving the main body of the ceramic artwork, in order to achieve the visual effect, and then to enrich The form and content of the work. Specific practices include styling tearing, dry folding, scratching, surface chapping and sanding. In addition, due to the characteristics and artistry of ceramic materials, the modeling process cannot be too complicated, and the principles of production design should be as simple and generous as possible. The former can be made using the design methods of yang, intaglio, hollow, stacking, tapping, bending, folding, bonding, squeezing, imprinting, etc. to win the texture. The latter can use points and lines. The combination of faces forms a variety of ceramic decorative shapes, creating a new style. This is the emotional expression of the designer. It is also the only way for them to pursue their individuality. Making texture is an important method in the creation of ceramic decoration, which can bring the work closer to the aesthetics. 


\section{Conclusion}

The ceramic texture has a wide range of performance neighborhoods and a wealth of potential. It can be called a piano in the construction of environmental art. It can be gorgeous or elegant, it can be simple and crystal-clear, and the artistic effect it produces can be either "to the masses" or "subtleties." But despite their dedication to such abundance, their inherent qualities and potentials have not been valued and developed. New environments, new ideas, and new requirements are forcing us to reflect on traditional old ideas.

\section{References}

[1] Men Fu Zhenyu. Correspondence Art Design (Public Art Design) Professional and Professional Curriculum Thinking [J]. Harbin Vocational Technical College Journal, 20 Sichuan (0 4): 021103.

[2] Wu Tianlin. On the decoration of ceramic art in China [J]. Chinese pottery porcelain, 2007, 43 (04): 66a67.

[3] Xi Wangzhong. A brief analysis of the decoration and ceramic art [J]. Chinese pottery porcelain, 2002, 38 (01): 56a57

[4] Wang Da, et al. University Sports and Health Course [M]. Peking University Press, 2001.

[5] Ji Liu, Other Education and Health [M]. East China Normal University Press, 2001. 